\title{
Analyses of venom spitting in African cobras (Elapidae: Serpentes)
}

\author{
Bruce A. Young ${ }^{1 *} \&$ Mark O'Shea ${ }^{2^{\dagger}}$ \\ ${ }^{1}$ School of Biological Sciences, Washington State University, P.O. Box 644236, Pullman, WA 99164-4236, U.S.A. \\ ${ }^{2}$ School of Applied Sciences, University of Wolverhampton, Wolverhampton, WV1 1SB, U.K.
}

Received 21 January 2004. Accepted 27 July 2004

\begin{abstract}
The venom spat by four spitting cobras (Naja nigricollis, N. pallida, N. mossambica and Hemachatus haemachatus) was caught using perspex plates. Densiometric analysis of the spat venom revealed low levels of variation in volume among successive spits. The dispersal patterns formed by the spat venom were divided into two basic classes, both of which were produced by all four species. The low levels of variation in venom volume, coupled with the variation in venom dispersal pattern, suggests a complexity to the regulation of venom flow in spitting cobras beyond simply neuromuscular control of the extrinsic venom gland.
\end{abstract}

Key words: defensive behaviour, snake, teeth, Naja, Hemachatus.

\section{INTRODUCTION}

Rosenberg (1967) described the basic mechanics of venom expulsion in snakes, emphasizing the role of the extrinsic venom gland musculature in creating the propulsive venom pressure. Young et al. (2000) supported Rosenberg's model by demonstrating a relationship between the contractile state of the extrinsic venom gland musculature and venom pressure in Crotalus atrox. Additional experimental analyses of crotalids demonstrated that the soft tissues of the fang sheath play a functional role in venom delivery (Young et al. 2001a). This led to the formulation of a new model, the pressure-balance model, for venom injection in snakes. This model postulates that the rate and volume of venom expulsion will be significantly influenced by the mutual interaction of venom pressure within the venom gland, venom pressure within soft-tissue chambers in the fang sheath, and peripheral resistance due to the target's tissue, and that these mutual interactions are dynamic due to the physical interactions between the snake and the target during fang penetration (Young et al. 2002). Though supported by experimental evidence (Young et al. 2003), it remains to be seen how broadly applicable this new model of venom injection is.

The phylogeny of snakes remains unresolved, yet there is a general consensus that a venom delivery system has evolved independently in multiple ophidian lineages (e.g. Cundall \& Greene 2000; Jackson 2003). There is some evidence of func-

*Author for correspondence: Email: youngb@wsu.edu tional convergence within these venom delivery systems; the fang sheath appears to play the same functional role in spitting cobras as it does in the independently evolved Crotalus (Young et al. 2004). The ability to 'spit' venom has evolved in at least three elapid radiations (e.g. Wüster 1996; Slowinski et al. 1997; Keogh 1998). There is interspecific variation in the spitting behaviour (Rassmussen et al. 1995); nevertheless, all spitting cobras have the ability to expel pressurized venom without making physical contact with their target.

Spitting cobras are an ideal study group for exploring the mechanics of venom expulsion since the complicating physical interactions with the target are absent from the spat venom. The recent model of venom injection (Young et al. 2002) proposed that the physical interaction between the target and the soft tissues of the snake may significantly influence the kinematics and volume of venom flow. Since these physical interactions are absent in spitting cobras, we hypothesize that there is less volumetric variation in spat than in injected venom. In this contribution we test this hypothesis by catching the spat venom of wild cobras and quantifying the venom volume. The terminal portion of the venom delivery system, the fang, is static, and its influence on the dispersal pattern of the spat venom is thus constant. Accordingly we hypothesize that the dispersal pattern of the spat venom should be relatively constant, at least at the level of an individual fang. We test this hypothesis by photographing and analysing the dispersal patterns of caught spat venom. 


\section{MATERIALS \& METHODS}

\section{Field studies}

Field trials were conducted in KwaZulu-Natal, South Africa. These trials involved 10 specimens of Naja mossambica (mean snout-vent length = $90.1 \mathrm{~cm}$, range $=66-102 \mathrm{~cm})$, and two specimens of Hemachatus haemachatus (svl = 94 and $96 \mathrm{~cm})$. All specimens were wild-caught. The trials were conducted outdoors in late morning in a place sheltered from the wind. Each specimen was placed on top of a portable table and induced to spit at the face of one of the authors. When approaching the cobra in a crouched posture, each author held a perspex plate $(28 \times 28 \mathrm{~cm}, 3 \mathrm{~mm}$ thick $)$ in front of his face. While holding the plate relatively still roughly $40 \mathrm{~cm}$ away from the cobra, the authors performed rapid lateral and forward movements of their head and upper body. These jerky movements provoked the spitting behaviour, and the spat venom was caught on the perspex plates. Each plate was labelled to establish the orientation of the venom dispersal pattern; by laying out a number of plates in advance, it was possible to subject the cobra to relatively constant levels of stimulation.

The highly viscous venom dried almost immediately upon contact with the perspex, making the dispersal pattern easy to see. In cases where not all of the venom was collected on the perspex plate, the trial was excluded from analysis. Four or more samples of spat venom were collected from each snake; in most cases the spitting episodes were consecutive - the only exceptions were cases where the spat venom missed the plate or was discharged well beyond the $40 \mathrm{~cm}$ range. The spitting episodes of each specimen that were used for analysis were all collected within a five-minute time interval, during which the snake was subject to a fairly constant level of stimulation. After the last spat venom was collected the snake was briefly restrained and its snout-vent length measured.

\section{Laboratory studies}

At Lafayette College additional spat venom was collected from a $67 \mathrm{~cm}$ svl specimen of Naja nigricollis as well as a $104 \mathrm{~cm}$ specimen of Naja pallida. These snakes were obtained commercially, and were housed in individual terraria with a 12:12 photoperiod, a temperature range of $26-31^{\circ} \mathrm{C}$, water ad libitum and a diet of pre-killed mice. Though performed indoors, the data collection procedure was identical to that used in the field trials. All animal maintenance and experimental procedures comply with existing guidelines for both live reptiles and venomous snakes, and were approved by the Lafayette College Institutional Animal Care and Use Committee.

\section{Data analysis}

A portable light chamber was constructed such that the venom dispersal pattern on each perspex plate could be documented using an EOS-10 digital camera (Canon) coupled to a G4 powerbook (Apple). At Lafayette College each perspex plate was positioned within an Alpha Imagen 8800 imaging system (Alpha Innotech, San Leandra, CA). This system created a digital image of the plate and then performed densiometric analysis of the spat venom. The software quantified the area of each venom droplet as well as its relative density; multiplying these values together, and summing every droplet from each spitting episode, provided a relative measure of the volume of spat venom.

We sought to compare variation in the volume of sequential spits from single specimens, rather than venom amount between specimens. To do this we defined the integrated densiometric value for the first sample of spat venom as being equal to 1.0 , and then examined the range of densiometric values for the subsequent spits. Where possible, the right and left fangs were quantified separately; however, all analyses were performed on total spat venom.

\section{RESULTS}

\section{Venom quantification}

The integrated densiometric values of the spat venom revealed no obvious pattern among successive spits (Fig. 1). The best fit linear regression line $\left(b=0.014, r^{2}=0.008\right)$ through these data had a coefficient which was not significantly different from $0(t=0.69, P=0.49)$. The same results were found in each individual snake and/or individual fang (Fig. 2); the variation in integrated venom density did not follow any obvious pattern.

The magnitude of change in venom volume during the spit sequence is simply the ratio between the largest and smallest total integrated venom densiometric values. The mean value for all the cobras examined was 1.77 (S.E. = 0.12; range $=1.30-2.81$ ). For the majority of cobras examined the amount of spat venom varied by a factor of less than 2 . 


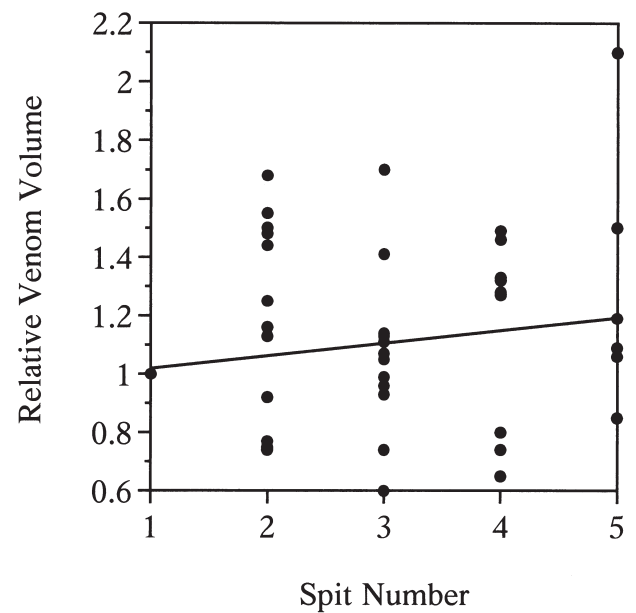

Fig. 1. Magnitude of total venom volume (both fangs) during successive spits from all specimens. The volume of the first spat is defined as 1.0 for each specimen.

\section{Pattern of venom dispersal}

Two classes of venom dispersal patterns were evident on the perspex plates. In the first class, present in $43 / 78$ or $55 \%$ of the trials, the venom expulsed from the right and left fangs remained distinct, the spat venom formed a geometric pattern on the plate, and the onset had greater density and volume (Fig. 3A). In the second class, which comprised $35 / 78$ or $45 \%$ of the trials, the spat venom was present as a single, normally oblate concretion (Fig. 3B). In dispersal patterns of the second class neither the individual contributions of the right and left fang, nor the onset of the spitting, could be determined.

Both types of dispersal patterns were produced by all species tested. There was considerable variation in the dispersal patterns produced by each specimen (Fig. 4). These variations included

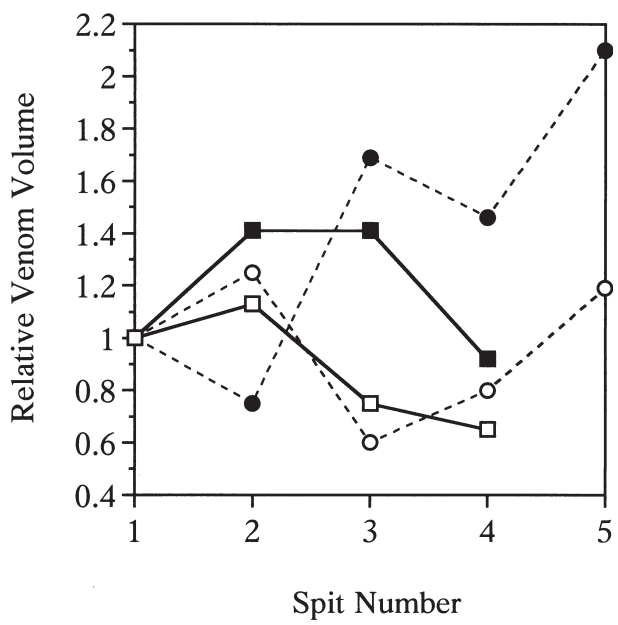

Fig. 2. Magnitude of venom volume during successive spits from a $98 \mathrm{~cm}$ svl specimen of Naja mossambica (squares) and a $67 \mathrm{~cm}$ svl Naja nigricollis (circles); open symbols are from the right fang, closed symbols are from the left fang. Note the absence of an obvious pattern in the venom volumes.

both classes of dispersal pattern, as well as markedly different geometric patterns. Many of the spitting episodes resulted in strongly arced or circular patterns, while others were more vertical or horizontal. The right and left fang always formed the same basic dispersal pattern. There was no relationship evident between the dispersal pattern and the temporal sequence of the spit. One specimen produced three distinct venom streams with each spitting episode, presumably reflecting a functional replacement fang as well as a soon to be shed fang on the right side of the head (Fig. 5). In each of these triple stream spits, the sum of the amount of spat venom from the two fangs on the right side was similar to that from the single fang on the left.
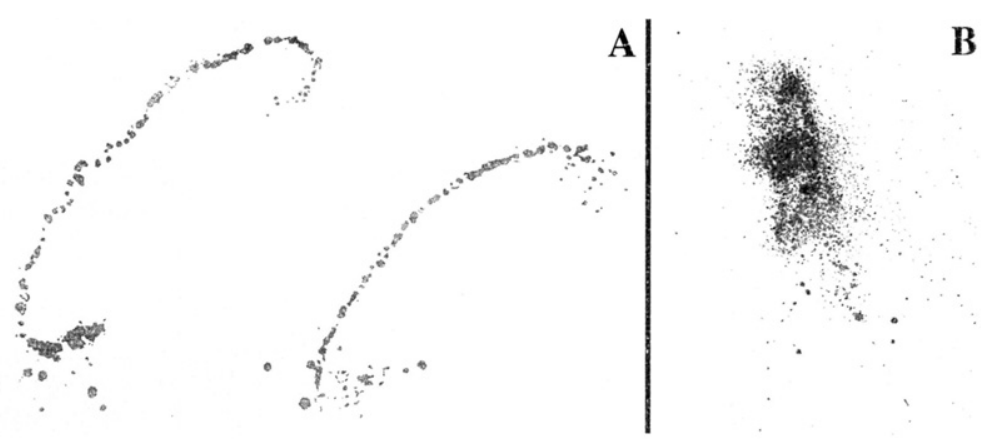

Fig. 3. Dispersal patterns of two spats from Naja mossambica. A, The first, or geometric, class of dispersal pattern; note the distinct traces of the two fangs, the arching pattern, and the greater volume at onset (bottom); B, the second, or concretion, class of dispersal pattern characterized by a single oblong venom pattern. 
A
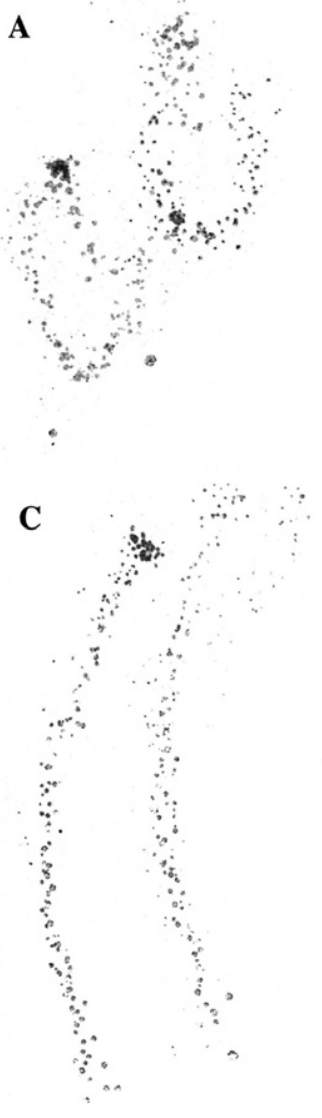

Fig. 4. Variation in the venom dispersal patterns. These four samples (A-D) are from successive spitting episodes of a single $85 \mathrm{~cm}$ svl specimen of Naja mossambica.

\section{DISCUSSION}

Quantification of the venom volume revealed that every specimen examined spat a rather consistent volume (mean magnitude of change was only 1.77). This is in agreement with an earlier study of Naja pallida which found that the dry mass of venom protein spat showed no significant difference between the 1st and the 20th spat, though it did become more variable by the 40th spat (Cascardi et al. 1999). By contrast, the magnitude of variation in the amount of venom injected during defensive or predatory bites is much higher, in some cases exceeding factors of 100 (magnitudes determined by dividing the maximum and minimum values presented by Hayes et al. 2002). As such, the findings of our study support the hypothesis that there is less variation in spat venom than in

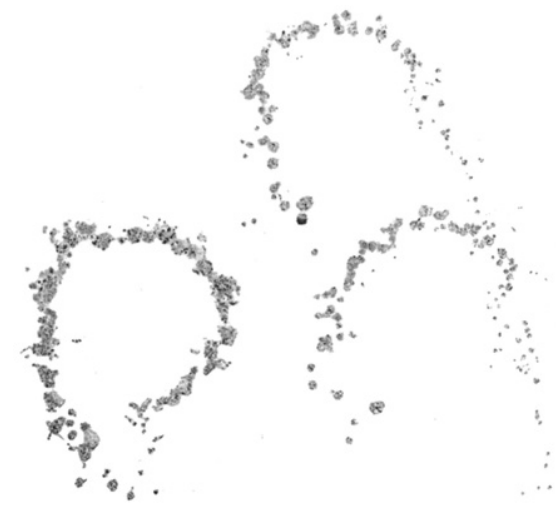

Fig. 5. Single spit from a $98 \mathrm{~cm}$ svl specimen of Naja mossambica showing two venom streams released from the right side of the snake, presumably reflecting discharge from both the functional and replacement fangs.

injected venom.

Hayes et al. (2002) postulated that the functional control of the extrinsic venom gland musculature differed between biting and spitting in cobras. The recent experimental analysis of spitting in cobras (Young et al. 2004) revealed that every spit was associated with active fang sheath deformation and active contraction of the extrinsic venom gland musculature. Combined EMG and kinematic analyses revealed that it was the deformation of the fang sheath, not the contraction of the extrinsic venom gland musculature, that regulated venom discharge during spitting (Young et al. 2004). Earlier study of venom regulation in Crotalus showed that the extrinsic venom gland musculature appeared to be compartmentalized, suggesting that these vipers had fine neural control over the extrinsic force applied to the venom gland. Our results suggest that, at least in spitting cobras, the fang sheath has a significant influence on venom expulsion.

The relative constancy of the volume of spat venom is in sharp contrast to the diversity of dispersal patterns (Fig. 4). The geometric patterns evident in the venom dispersal patterns (Figs 3-5) may reflect slight movements of the head during venom expulsion. Young et al. (2004) recorded the spitting behaviour using high-speed digital video and noted regular movements of the head and venom stream. The presence of two different classes of dispersal patterns was unexpected, and contrary to our hypothesis. The single concretions may represent experimental errors in which the perspex plate was held too close to the cobra, such that the spat venom was collected before it could 
disperse. We do not believe such a systemic error occurred during data collection, and we have frequently witnessed geometric dispersal patterns in spat venom caught at distances under $40 \mathrm{~cm}$ (such as when captive specimens spit against the glass of their enclosure). We hypothesize that these different classes of dispersal patterns may reflect different flow regimes of venom through the venom delivery system. Given the relative constancy of venom volumes, and the fixed nature of the fang and exit orifice, these different flow regimes could most easily be produced as the venom courses through the soft tissue chambers located within the fang sheath (Young et al. 2001b). The fact that diverse dispersal patterns were found in each species is particularly surprising, since these species have markedly different spitting behaviours (Rasmussen et al. 1995).

The venom of spitting cobras is often described as dilute (e.g. Branch 1997) which has led to some speculation that the fluid spat by spitting cobras is a combination of venom and saliva, or that cobras can switch from spitting venom to spitting saliva (e.g. Rose 1962). Though spatially close, there is no functional connection between the venom delivery system and the supralabial gland (the site of saliva production) in cobras (e.g. Taub 1966; Kochva 1978). The secretory product of the supralabial gland is drained by a series of small ductules; these ductules do not approach the entrance orifice of the fang, and there is no obvious mechanism which would expel this secretory product under high pressure. The low variation in volume of spat venom measured in our study, and the relatively consistent dried masses of spat venom determined in the earlier study (Cascardi et al. 1999) further argue against a dynamic combination of two secretory products.

Venom spitting is used exclusively as a defence. The venom has little or no effect on unbroken skin, but even small quantities of the venom can produce significant damage, including permanent blindness, to corneas (e.g. Warrell \& Ormerod 1976; Ismail et al. 1993). Despite this, there is no experimental evidence regarding the accuracy of spitting cobras, or even if they can recognize and preferentially target eyes, as has been shown in other snakes (e.g. Herzog \& Bern 1992). The cepahlic movements described during venom spitting (Young et al. 2004), which reflect the contraction of skeletal muscles associated with venom expulsion, may also function to increase the dispersal area of the spat venom thereby maximizing the chance of making contact with the cornea.

\section{ACKNOWLEDGEMENTS}

The authors wish to thank Donald Strydom and Warren Klein (Swadini Reptile Park, Limpopo Province) for their assistance with the cobras, as well as Pine Pienaar and the staff of Bankhoek Lodge, Mkuze, for their hospitality. This research was supported by YAP Films (Leeds and Toronto) and Animal Planet (Washington) and by NSF grant IBN-0129805 to B.A.Y.

\section{REFERENCES}

BRANCH, B. 1997. Field Guide to the Snakes and other Reptiles of Southern Africa. Ralph Curtis, Florida.

CASCARDI, J., YOUNG, B.A., HUSIC, D. \& SHERMA, J. 1999. Protein variation in the venom spat by the pink spitting cobra (Naja pallida) (Reptilia: Serpentes). Toxicon 37: 1271-1279.

CUNDALL, D. \& GREENE, H.W. 2000. Feeding in snakes. In: Feeding: Form, Function and Evolution in Tetrapod Vertebrates, (ed.) K. Schwenk, pp. 293-333. Academic Press, New York.

HAYES, W.K., HERBERT, S.S., REHLING, G.C. \& GENNARO, J.F. 2002. Factors that influence venom expenditure by viperid and other snakes during predatory and defensive contexts. In: Biology of Vipers, (eds) G.W. Schuett, M. Hoggren, M.E. Douglas \& H.W. Greene, pp. 207-233. Eagle Mountain Publishing, Utah.

HERZOG, H.A. \& BERN, C. 1992. Do garter snakes strike at the eyes of predators? Animal Behaviour 44: 771773.

ISMAIL, M., AL-BEKAIRI, A., EL-BEDAIWY, A., \& ABD-EL SALAM, M. 1993. The ocular effects of spitting cobras: I. The ringhals cobra (Hemachatus haemachatus) venom-induced corneal opacification syndrome. Clinical Toxicology 31: 31-41.

JACKSON, K. 2003. The evolution of venom-delivery systems in snakes. Zoological Journal of the Linnean Society of London 137: 337-354.

KEOGH, J.S. 1998. Molecular phylogeny of elapid snakes and a consideration of their biogeographic history. Biological Journal of the Linnean Society of London 63: 177-203.

KOCHVA, E. 1978. Oral glands of the Reptilia. In: Biology of the Reptiles, (eds) C. Gans \& K. Gans, vol. 10, pp. 43-161. Academic Press, New York.

RASMUSSEN, S., YOUNG, B.A. \& KRIMM, H. 1995. On the 'spitting' behaviour in cobras. Journal of Zoology, London 237: 27-36.

ROSE, W. 1962. The Reptiles and Amphibians of Southern Africa. Maskew Miller, Cape Town.

ROSENBERG, H. 1967. Histology, histochemistry and emptying mechanism of the venom gland of some elapid snakes. Journal of Morphology 122: 133-156.

SLOWINSKI, J., KNIGHT, A. \& ROONEY, A. 1997. Inferring species trees from gene trees: a phylogenetic analysis of the Elapidae (Serpentes) based on the amino acid sequences of venom protein. Molecular 
Phylogeny and Evolution 8: 349-362.

TAUB, A.M. 1966. Ophidian cephalic glands. Journal of Morphology 118: 529-542.

WARRELL, D. \& ORMEROD, L. 1976. Snake venom ophthalmia and blindness caused by the spitting cobra (Naja nigricollis) in Nigeria. American Journal of Tropical Medicine and Hygiene 25: 525-529.

WÜSTER, W. 1996. Taxonomic changes and toxinology: systematic revisions of the Asiatic cobras (Naja naja species complex). Toxicon 34: 399-406.

YOUNG, B.A., ZAHN, K., BLAIR, M. \& LALOR, J. 2000. Functional subdivision of the venom gland musculature and the regulation of venom expulsion in rattlesnakes. Journal of Morphology 246: 249-259.

YOUNG, B.A., BLAIR, M., ZAHN, K. \& MARVIN, J. 2001a. Mechanics of venom expulsion in Crotalus, with special reference to the role of the fang sheath. Anatomical Record 264: 415-426.

YOUNG, B.A., DALEY, K. \& LEE, C. 2001b. The comparative anatomy of the fang sheath in snakes. American Zoologist 41: 1633 (Abstract).

YOUNG, B.A., LEE, C. \& DALEY, K. 2002. Do snakes meter venom? Biosciences 52: 1121-1126.

YOUNG, B.A., PHELAN, M., MORAIN, M., OMMUNDSEN, M. \& KURT, R. 2003. Venom injection in rattlesnakes (Crotalus): peripheral resistance and the pressure-balance hypothesis. Canadian Journal of Zoology 81: 313-320.

YOUNG, B.A., DUNLAP, K., KOENIG, K. \& SINGER, M. 2004. The buccal buckle: the functional morphology of venom spitting in cobras. Journal of Experimental Biology Biology 207: 3483-3494. 